\title{
Ressonância magnética para avaliação de tumores malignos da cavidade oral: uma revisão da literatura
}

\author{
- Isabela Goulart Gil Choi Departamento de Estomatologia, Faculdade de Odontologia, Universidade de São Paulo, \\ SP, Brasil - Moses Park Departamento de Estomatologia, Faculdade de Odontologia, Universidade de São Paulo, SP, \\ Brasil - Fernando Antonio Reis Laurino Departamento de Estomatologia, Faculdade de Odontologia, Universidade de \\ São Paulo, SP, Brasil • Arthur Rodriguez Gonzalez Cortes Departamento de Estomatologia, Faculdade de Odontologia, \\ Universidade de São Paulo, SP, Brasil • Emiko Saito Arita Departamento de Estomatologia, Faculdade de Odontologia, \\ Universidade de São Paulo, SP, Brasil
}

RESUMO || Objetivo: Realizar uma revisão de literatura sobre o desempenho das imagens de ressonância magnética (RM) para avaliação de tumores malignos da cavidade bucal. Métodos: Os bancos de dados MEDLINE (via PubMed) e EMBASE foram utilizados para pesquisar artigos publicados até 2017, restritos a publicações em inglês e em português. As listas de referências de quaisquer artigos em potencial e o banco de dados OpenGrey também foram avaliados para encontrar estudos ou documentos não publicados ou que não foram identificados pela pesquisa eletrônica. O critério de inclusão foi: as pesquisas serem artigos originais, revisões sistemáticas e/ou relatos de casos que discutissem sobre o uso de imagens de RM na avaliação de tumores malignos da cavidade oral. A revisão da literatura foi realizada de acordo com as diretrizes do PRISMA. Resultados: Um total de 25 estudos foram identificados como elegíveis para inclusão nesta revisão e agrupados para discussão. Os tumores malignos abordados nestes estudos foram: carcinoma de células escamosas, tumores das glândulas salivares, sarcomas orais e maxilofaciais, e melanomas. Conclusão: A RM mostrou-se uma técnica pré-operatória muito precisa para a avaliação de tumores malignos da cavidade bucal.

DESCRITORES | Glândulas Salivares; Imagem por Ressonância Magnética; Imagem Diagnóstica; Tumor da Glândula Salivar; Carcinoma de Células Escamosas.

ABSTRACT | Magnetic resonance imaging for assessing malignant tumors in the oral cavity: a literature review • Objectives: To perform a literature review to assess MRI performance as a diagnostic auxiliary imaging technique for evaluation of malignant tumors in the oral cavity. Methods: The MEDLINE (through PubMed) and EMBASE databases were searched for articles published up to 2017, restricted to English and Portuguese publications. Reference lists of any potential articles and the OpenGrey database were also evaluated to find potential unpublished studies or papers that were not identified by electronic search. The inclusion criteria encompassed original articles, systematic reviews, and case reports for the analysis of MRI images in the evaluation of oral cavity malignant tumors. The literature review was performed according to the PRISMA guidelines. Results: A total of 25 studies were considered eligible for inclusion in this literature review and assembled for discussion. The malignant tumors approached in these studies were: Squamous cell carcinoma, salivary gland tumors, oral and maxillofacial sarcomas, and melanomas. Conclusion: The MRI has shown itself to be a very precise preoperatory technique for assessing malignant tumors in the oral cavity. 


\section{INTRODUÇÃO}

Ao existir uma suspeita da presença de tumor maligno na cavidade oral, a avaliação do tipo, tamanho, extensão e padrão da disseminação desse tumor é necessária para os profissionais traçarem o melhor plano de tratamento possível. O diagnóstico pré-operatório consiste na combinação de avaliação física, exames complementares de imagem e biópsias locais. Um dos aspectos mais importantes dessa avaliação é determinar o grau de invasão óssea local da malignidade, uma vez que a extensão do procedimento cirúrgico e a aplicação das modalidades terapêuticas são influenciadas por essa informação. ${ }^{1}$

Diferentes recursos de imagem, como a tomografia computadorizada (TC), a ressonância magnética (RM) e a tomografia computadorizada por emissão de pósitrons (PET-CT), estão disponíveis para auxiliar no diagnóstico. A discussão para a escolha da modalidade ideal para avaliação dos tumores malignos ainda é controversa e continua em andamento, uma vez que cada técnica de imagem possui vantagens distintas para a obtenção dos resultados sobre o estadiamento do tumor. ${ }^{2}$

A cavidade oral está entre os dez sítios mais comuns para ocorrência de câncer no mundo. Estudos têm demonstrado que a extensão e a propagação do tumor medida com RM se correlacionam diretamente com os achados histológicos, sendo uma técnica muito eficiente para guiar as cirurgias ressectivas de tumor. A imagem de RM permite a determinação do grau de infiltração do tumor no osso esponjoso e na extensão extra-alveolar. ${ }^{3-5}$

\section{MATERIAIS E MÉTODOS}

Uma revisão sistemática foi feita para avaliar o desempenho da RM como técnica de imagem auxiliar para avaliação de tumores malignos que ocorrem na cavidade oral. Os bancos de dados MEDLINE (via PubMed) e EMBASE foram utilizados para pesquisar artigos publicados até 2017, restritos a publicações em inglês e português. A estratégia de pesquisa desenvolvida para a MEDLINE foi: "malignant tumors MRI OR squamous cell carcinoma AND MRI OR salivary gland tumor AND MRI OR malignant melanoma oral AND MRI OR melanoma oral AND MRI”. As referências de quaisquer artigos e o banco de dados OpenGrey também foram avaliados para identificação de possíveis estudos ou documentos não publicados ou que não foram identificados pela pesquisa eletrônica. O critérios de inclusão foi: as pesquisas serem artigos originais, revisões sistemáticas ou relatos de casos que discorriam sobre o uso da RM para avaliação de tumores malignos na cavidade oral. O texto foi estruturado de acordo com as diretrizes do PRISMA (Preferred Reporting Items for Systematic Reviews and Meta-Analyses). ${ }^{6}$

\section{Extração dos dados}

Três revisores independentes com experiência em radiologia examinaram os títulos, resumos e textos completos dos artigos identificados. Os seguintes dados sobre os artigos foram extraídos e registrados: ano de publicação, localização do ensaio, características dos grupos ou da amostra, características metodológicas, resultados, conclusões e fonte de financiamento (se aplicável).

\section{RESULTADOS}

Um total de 577 artigos foi inicialmente selecionado. Destes, 498 foram excluídos após avaliação do título, resumo ou palavras-chave, resultando em 79 artigos que potencialmente atendia o critério de inclusão. Em seguida, um total de 54 estudos foram excluídos, pelo fato de seus textos completos não esclarecerem o papel da RM para avaliação dos tumores malignos na cavidade oral. Como resultado, um total de 25 estudos foi finalmente identificado como elegível para inclusão nesta revisão sistemática e agrupado para discussão. 


\section{Discussão}

\section{Carcinoma epidermoide}

O carcinoma epidermoide é a neoplasia primária mais frequente de origem epitelial e o câncer mais comum da cavidade oral. A extensão do tumor, possíveis metástases nodais e a propagação extracapsular são todos fatores que afetam o tratamento do paciente e prognóstico. ${ }^{3,5}$

O carcinoma epidermoide acomete principalmente a língua, a base da língua e as tonsilas. ${ }^{4}$ Normalmente, o tamanho e profundidade dos tumores são avaliados utilizando os métodos de imagem de TC ou RM. No entanto, a RM é amplamente aceita como uma técnica superior à TC para avaliação de lesões de tecidos moles. A intensidade média de invasão determinada em espécimes histológicos tem se mostrado menor do que a intensidade de invasão determinada por imagens de RM, provavelmente devido ao encolhimento tecidual durante a preparação das lâminas. ${ }^{5,7,8}$
Estudos têm mostrado que imagens ponderadas em T1 de RM de 1,5 tesla são mais precisas para avaliação da profundidade de invasão e estadiamento do tumor para o planejamento cirúrgico. Aparentemente, as imagens ponderadas em T2 são superestimadas devido à inflamação e edema nos tecidos circundantes ao tumor. ${ }^{3,5,8}$ No entanto, em um estudo realizado utilizando RM de 3 tesla (imagens de eco gradiente) para avaliação do carcinoma epidermoide em língua de 25 pacientes mostrou que as medidas obtidas se correlacionaram com os achados histológicos e que essa técnica de imagem é mais efetiva para detecção de adenopatia maligna com disseminação extracapsular. Os autores concluíram que a $\mathrm{RM}$ de 3 tesla apresenta maior sensibilidade, especificidade e precisão na detecção de metástases em linfonodos quando comparada com outros estudos publicados utilizando RM de 1,5 tesla. As imagens obtidas com o aparelho de 3 tesla estão ilustradas na Figura $1 .{ }^{9}$
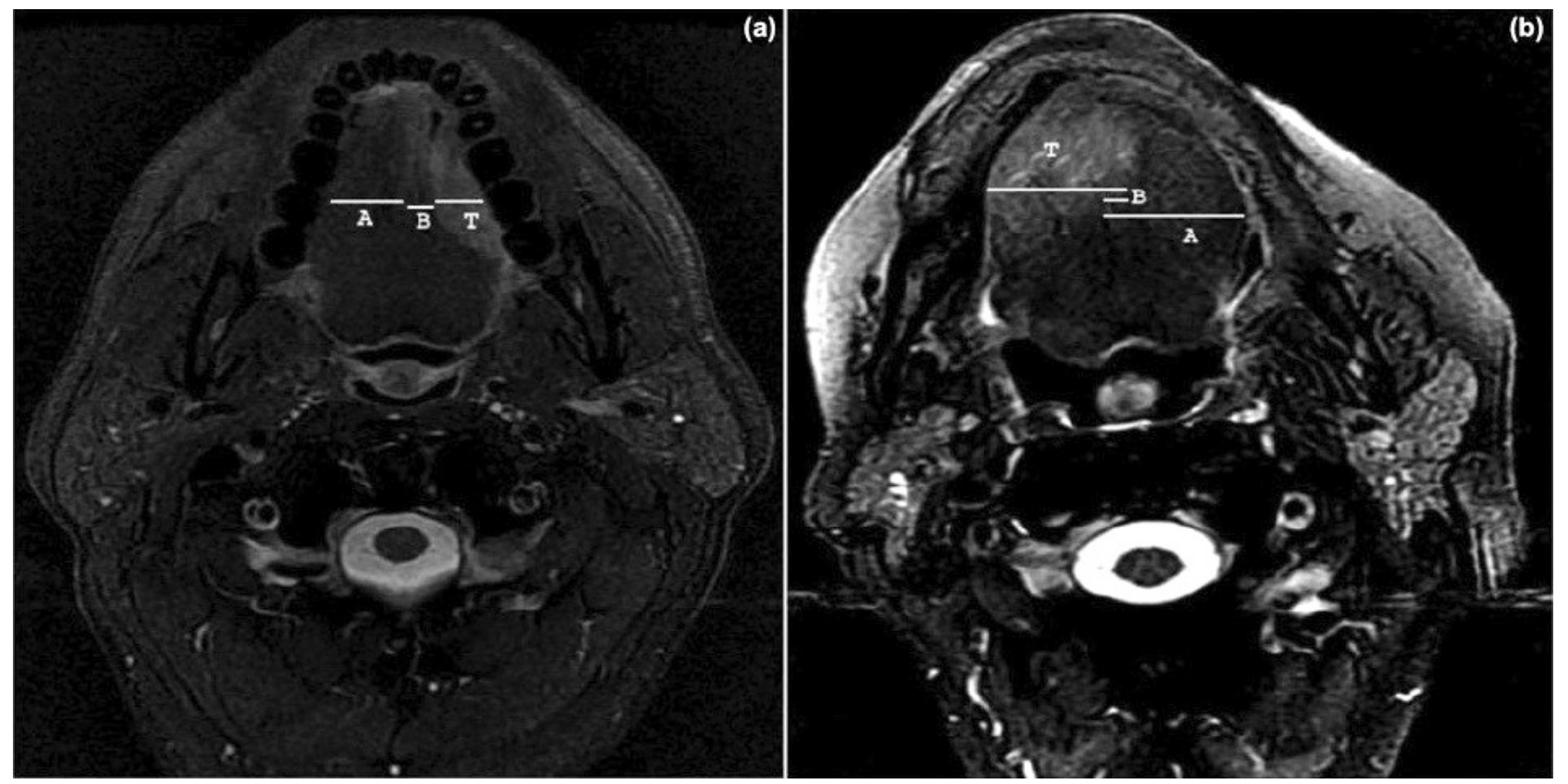

Figura 1 || Imagem de RM obtida em aparelho de 3 tesla mostrando medidas. Em (a) o tumor não se estende além da linha média; em (b) o tumor se estende além da linha média (tumor em língua profundamente invasivo). T: espessura, a distância da superfície do tumor até o ponto mais profundo da invasão tumoral; A: a distância da superfície ao septo lingual no lado não afetado; B: a distância do septo ao ponto de invasão tumoral mais profunda. A espessura em imagem de ressonância magnética reconstruída é igual a A-B (a) e A+B (b). Imagem obtida do artigo de Moreno KF, et al. ${ }^{9}$ 
Em outro estudo, pesquisadores avaliaram o valor diagnóstico adicional oferecido pelas imagens em difusão de RM para avaliação da malignidade residual de carcinomas epidermoides após tratamento com radioterapia. Eles concluíram que a combinação de imagens RM com as imagens de tomografia de emissão de pósitrons (PET-CT com 18F-FDG), que é a técnica frequentemente utilizada para detectar malignidade residual de carcinomas epidermoides da região de cabeça e pescoço, ofereceu uma maior sensibilidade e especificidade, sugerindo que as duas técnicas possam ser complementares para detecção loco-regional de malignidade residual. ${ }^{10}$

\section{Tumores de glândulas salivares}

Os tumores de glândulas salivares representam cerca de $5 \%$ de todos os tumores presentes em região de cabeça e pescoço, sendo a maioria benignos. ${ }^{11,12}$ O carcinoma mucoepidermoide é o tumor maligno mais comum que acomete as glândulas salivares, principalmente as parótidas. ${ }^{13}$

A RM apresenta vantagens sobre a TC para avaliação dos tumores de glândulas salivares, pois tem excelente resolução de partes moles e acurácia na delimitação da extensão tumoral (sendo indicada para o planejamento cirúrgico) e permite avaliação apropriada da musculatura extrínseca da língua e das glândulas salivares menores, sublinguais e das vias de disseminação tumoral. ${ }^{14-16}$

Para o diagnóstico de tumores malignos em glândulas salivares, vários estudos relataram a efetividade de imagens de RM, particularmente de imagens com contraste e ponderadas por difusão. ${ }^{17-19}$, sendo a imagem ponderada em difusão o protocolo de RM que fornece informações quantitativas referentes à magnitude e direção da difusão da água no espaço tridimensional. Esse grau de difusão pode ser quantificado e é denominado de coeficiente de difusão aparente (ADC). ${ }^{20}$

A medição do ADC tem se mostrado útil para diferenciar tumores benignos e malignos presentes em glândulas salivares. ${ }^{19,21}$ No entanto, essa diferenciação é limitada devido à existência de muitos tipos distintos de tumores, principalmente para distinguir o tumor de Warthin (tumor benigno de glândulas salivares) dos tumores malignos da glândula parótida. ${ }^{17,22} \mathrm{O}$ tumor de Warthin é conhecido por ter valores baixos de ADC em relação a outros tipos de tumores benignos presentes na glândula parótida. Portanto, diferenças estatisticamente significantes entre o tumor de Warthin e os tumores malignos não são obtidas. ${ }^{17,20,22}$

Um estudo propôs avaliar os valores de coeficiente de difusão e anisotropia fracionada (FA) - um índice quantitativo da anisotropia do tecido - para diferenciar tumores benignos e malignos. O estudo mostrou que os valores de FA em tumores malignos eram significativamente maiores do que todos os subtipos tumorais benignos (adenomas pleomórficos, tumores de Warthin e outros). A área sob a curva Receiver Operating Characteristic (ROC) do valor FA foi significativamente maior que a do valor de $\mathrm{ADC}$, portanto o estudo mostrou que valores de FA podem adicionar informações úteis na diferenciação entre tumores em glândulas parótidas. ${ }^{20}$

\section{Sarcomas da região oral e maxilofacial}

Os sarcomas de tecido mole englobam uma ampla gama de tumores malignos que surgem de tecidos mesenquimais em qualquer sítio anatômico. São considerados tumores raros; os tumores malignos que acometem a boca representam menos de $1 \%$. A literatura sobre sarcomas de tecido mole é escassa, com apenas alguns poucos relatos de caso clínico. ${ }^{23,24}$

Os sarcomas que se originam dos tecidos musculares lisos são conhecidos como leiomiossarcomas. Eles podem inicialmente aparecer nos tecidos gengivais como um aumento não ulcerado. Em um caso clínico reportado na literatura, uma mulher de 58 anos que apareceu com um aumento no lado esquerdo de sua mandíbula, sem dor, mas com dificuldade no encaixe de sua 
prótese na arcada inferior, foi diagnosticada com leiomiossarcoma com base na avaliação clínica, exame histológico e de imagens. A RM foi importante, uma vez que mostrou a perda óssea preenchida com um tumor de tamanho irregular, localizado nas regiões de ângulo e ramo da mandíbula e com uma destruição óssea cortical considerável. ${ }^{25}$

Já em relação aos osteossarcomas, estes representam cerca de 20\% dos tumores ósseos primários. Os sítios mais comuns para sua ocorrência são os ossos longos, sendo incomuns os tumores em maxilares. Quando ocorrem, as regiões de corpo e ângulo da mandíbula são as mais afetadas. ${ }^{26}$

Estudos têm mostrado que a RM é superior à TC na determinação de invasão medular óssea, extensão perineural e envolvimento de partes moles. No entanto, a TC é superior na avaliação de destruição óssea, erosão de cortical, reconstruções volumétricas $3 \mathrm{D}$ pré-operatórias, planejamento de implantes e reconstruções craniofaciais. Portanto, as duas técnicas de imagem devem ser vistas como complementares na avaliação desses tumores. ${ }^{14-16,27}$

\section{Melanomas}

O melanoma maligno de mucosa na cavidade oral é um tumor raro, representando apenas 0,2 a $8 \%$ de todos os melanomas malignos, mas possui uma tendência à metástase maior do que a de outros tipos de tumores malignos que ocorrem na boca. Normalmente aparece na região do palato ou no rebordo alveolar da maxila. ${ }^{28}$

Estudos têm demonstrado que melanomas na região de cabeça e pescoço reduzem os valores de $\mathrm{T} 1$ e T2 nas imagens de RM, devido aos radicais livres nos pigmentos de melanina serem paramagnéticos. Revelam ainda que esses tumores são caracterizados por imagens hiperintensas ponderadas em T1 e imagens intermediárias até hipointensas ponderadas em T2. ${ }^{26,29}$

Em um relato de caso clínico, imagens de RM em um aparelho de 1,5 tesla foram obtidas para um melanoma. O tumor foi demonstrado com mais clareza nas imagens saturadas em gordura do que nas imagens ponderadas em $\mathrm{T} 1$ convencionais. Como a medula óssea da mandíbula contém grandes quantidades de gordura, as imagens ponderadas em T1 pós-contraste e saturadas em gordura mostraram com maior precisão o contorno e extensão do tumor, e os achados imaginológicos foram precisamente compatíveis com o resultado histológico. ${ }^{28}$

\section{CONCLUSÃO}

A RM tem se mostrado uma técnica préoperatória muito precisa para avaliação dos tumores malignos na cavidade oral. Ela tem fornecido informações importantes, mas com protocolos distintos de imagem para cada tipo de tumor. A literatura sobre alguns tipos de tumores malignos na cavidade oral, como os sarcomas de tecido mole e os melanomas, ainda se encontra escassa. No entanto, os poucos relatos que existem sobre esses tipos de tumores deixam claro o valor da RM como método auxiliar no diagnóstico.

\section{REFERÊNCIAS}

1. Dreiseidler T, Alarabi N, Ritter L, Rothamel D, Scheer M, Zöller JE, et al. A comparison of multislice computerized tomography, cone-beam computerized tomography, and single photon emission computerized tomography for the assessment of bone invasion by oral malignancies. Oral Surg Oral Med Oral Pathol Oral Radiol Endod. 2011 Sep;112(3):367-74. doi: 10.1016/j.tripleo.2011.04.001 .

2. Alberico RA, Husain SHS, Sirotkin I. Imaging in head and neck oncology. Surg Oncol Clin N Am. 2004 Jan;13(1):13-35 doi: 10.1016/S1055-3207(03)o0124-8.

3. Lam P, Au-Yeung KM, Cheng PW, Wei WI, Yuen AP, Trendell-Smith N, et al. Correlating MRI and histologic tumor thickness in the assessment of oral tongue cancer. AJR Am J Roentgenol. 2004 Mar;182(3):803-8. doi: 10.2214/ ajr.182.3.1820803.

4. Park JO, Jung SL, Joo YH, Jung CK, Cho KJ, Kim MS. Diagnostic accuracy of magnetic resonance imaging (MRI) in the assessment of tumor invasion depth in oral/oropharyngeal 
cancer. Oral Oncol. 2011 May;47(5):381-6. doi: 10.1016/j.oraloncology.2011.03.012.

5. Preda L, Chiesa F, Calabrese L, Latronico A, Bruschini R, Leon ME, et al. Relationship between histologic thickness of tongue carcinoma and thickness estimated from preoperative MRI. Eur Radiol. 2006 Oct;16(10):2242-8. doi: 10.1007/ soo330-006-0263-9.

6. Moher D, Liberati A, Tetzlaff J, Altman DG, PRISMA Group. Preferred Reporting Items for Systematic Reviews and Meta-Analyses: the PRISMA statement. PLoS Med. 2009 Jul; 6(7):e1000097. doi: 10.1371/journal.pmed.1000097.

7. Bradley PJ, MacLennan K, Brakenhoff RH, Leemans CR. Status of primary tumour surgical margins in squamous head and neck cancer: prognostic implications. Curr Opin Otolaryngol Head Neck Surg. 2007 Apr;15(2):74-81. doi: 10.1097/MOO.obo13e32805867of.

8. Jung J, Cho NH, Kim J, Choi EC, Lee SY, Byeon HK, et al. Significant invasion depth of early oral tongue cancer originated from the lateral border to predict regional metastases and prognosis. Int J Oral Maxillofac Surg. 2009 Jun;38(6):6536o. doi: 10.1016/j.ijom.2009.01.004.

9. Moreno KF, Cornelius RS, Lucas FV, Meinzen-Derr J, Patil YJ. Using 3 Tesla magnetic resonance imaging in the pre-operative evaluation of tongue carcinoma. J Laryngol Otol. 2017 Sep;131(9):793-80o. doi: 10.1017/Soo2221511700136o.

10. Noij DP, Jagesar VA, Graaf P, Jong MC, Hoekstra OS, Bree

$\mathrm{R}$, et al. Detection of residual head and neck squamous cell carcinoma after (chemo)radiotherapy: a pilot study assessing the value of diffusion-weighted magnetic resonance imaging as an adjunct to PET-CT using 18F-FDG. Oral Surg Oral Med Oral Pathol Oral Radiol. 2017 Sep;124(3):296-305.e2. doi: 10.1016/j.00oo.2017.04.011.

11. Ho K, Lin H, Ann DK, Chu PG, Yen Y. An overview of the rare parotid gland cancer. Head Neck Oncol. 2011;3:40. doi: 10.1186/1758-3284-3-40.

12. Shishegar M, Ashraf MJ, Azarpira N, Khademi B, Hashemi B, Ashrafi A. Salivary gland tumors in maxillofacial region: a retrospective study of 130 cases in a southern Iranian population. Patholog Res Int. 2011;2011:934350. doi: 10.4061/2011/934350.

13. Fonseca FP, Carvalho MV, Almeida OP, Rangel AL, Takizawa MC, Bueno AG, et al. Clinicopathologic analysis of 493 cases of salivary gland tumors in a Southern Brazilian population. Oral Surg Oral Med Oral Pathol Oral Radiol. 2012 Aug;114(2):230-9. doi: 10.1016/j.oooo.2012.04.008.
14. Saindane AM. Pitfalls in the staging of cancer of thyroid. Neuroimaging Clin N Am. 2013 Feb;23(1):123-45. doi: 10.1016/j.nic.2012.08.010.

15. Singh A, Thukral CL, Gupta K, Sood AS, Singla H, Singh K. Role of MRI in evaluation of malignant lesions of tongue and oral cavity. Pol J Radiol. 2017;82:92-9. doi: 10.12659/PJR.899352 .

16. Trotta BM, Pease CS, Rasamny JJ, Raghavan P, Mukherjee S. Oral cavity and oropharyngeal squamous cell cancer: key imaging findings for staging and treatment planning. Radiographics. 2011 Mar-Apr;31(2):339-54. doi: 10.1148/rg.312105107.

17. Habermann CR, Arndt C, Graessner J, Diestel L, Petersen KU, Reitmeier F, et al. Diffusion-weighted echo-planar MR imaging of primary parotid gland tumors: is a prediction of different histologic subtypes possible? AJNR Am J Neuroradiol. 2009 Mar;30(3):591-6. doi: 10.3174/ajnr.A1412.

18. Yabuuchi H, Fukuya T, Tajima T, Hachitanda Y, Tomita K, Koga M. Salivary gland tumors: diagnostic value of gadolinium-enhanced dynamic MR imaging with histopathologic correlation. Radiology. 2003 Feb;226(2):345-54. doi: 10.1148/ radiol.2262011486.

19. Yabuuchi H, Matsuo Y, Kamitani T, Setoguchi T, Okafuji T, Soeda H, et al. Parotid gland tumors: can addition of diffusion-weighted MR imaging to dynamic contrast-enhanced MR imaging improve diagnostic accuracy in characterization? Radiology. 2008 Dec; 249(3):909-16. doi: 10.1148/ radiol.2493072045.

20. Takumi K, Fukukura Y, Hakamada H, Ideue J, Kumagae Y, Yoshiura T. Value of diffusion tensor imaging in differentiating malignant from benign parotid gland tumors. Eur J Radiol. 2017 Oct;95:249-56. doi: 10.1016/j. ejrad.2017.08.013.

21. Celebi I, Mahmutoglu AS, Ucgul A, Ulusay SM, Basak T, Basak M. Quantitative diffusion-weighted magnetic resonance imaging in the evaluation of parotid gland masses: a study with histopathological correlation. Clin Imaging. 2013 Mar-Apr;37(2):232-8. doi: 10.1016/j.clinimag.2012.04.025.

22. Matsushima N, Maeda M, Takamura M, Takeda K. Apparent diffusion coefficients of benign and malignant salivary gland tumors: comparison to histopathological findings. J Neuroradiol. 2007 Jul;34(3):183-9. doi: 10.1016/j.neurad.2007.04.002.

23. Dito WR, Batsakis JG. Rhabdomyosarcoma of the head and neck: an appraisal of the biologic behavior in 170 cases. Arch Surg. 1962 May;84(5):582-8. doi: 10.1001/archsurg.1962.01300230098020. 
24. Yamamoto H, Kozawa Y, Takagi M, Otake S. Rhabdomyosarcoma of the left mandible. J Oral Maxillofac Surg. 1984 Sep;42(9):613-8. doi: 10.1016/0278-2391(84)90094-6.

25. Lewandowski B, Brodowski R, Pakla P, Stopyra W, Gawron I. Leiomyosarcoma in the mandible: a rare case report. Medicine (Baltimore). 2016 Jul;95(27):e4011. doi: 10.1097/ MD.0000000000004011.

26. Uchiyama Y, Matsumoto K, Murakami S, Kanesaki T, Matsumoto A, Kishino M, et al. MRI in a case of osteosarcoma in the temporomandibular joint. Dentomaxillofac Radiol. 2014 Feb;43(2):20130280. doi: 10.1259/dmfr.20130280.
27. Law CP, Chandra RV, Hoang JK, Phal PM. Imaging the oral cavity: key concepts for the radiologist. Br J Radiol. 2011 Oct;84(1006):944-57. doi: 10.1259/bjr/70520972.

28. Manigandan T, Sagar GV, Amudhan A, Hemalatha VT, Babu NA. Oral malignant melanoma: a case report with review of literature. Contemp Clin Dent. 2014 Jul-Sep;5(3):415-8. doi: 10.4103/0976-237X.137978.

29. Woodruff WW Jr, Djang WT, McLendon RE, Heinz ER, Voorhees DR. Intracerebral malignant melanoma: high-field-strength MR imaging. Radiology. 1987 Oct;165(1):209-13. doi: 10.1148/radiology.165.1.3628773. 